\title{
Levitation and percolation in quantum Hall systems with correlated disorder
}

\author{
Hui Song, ${ }^{1}$ Isao Maruyama, ${ }^{1}$ and Yasuhiro Hatsugai ${ }^{1,2, *}$ \\ ${ }^{1}$ Department of Applied Physics, University of Tokyo 7-3-1 Hongo, Bunkyo-ku, Tokyo 113-8656, Japan \\ ${ }^{2}$ Institute of Physics, University of Tsukuba 1-1-1 Tennodai, Tukuba, Ibaraki 305-8571, Japan \\ (Received 11 May 2007; revised manuscript received 8 August 2007; published 12 October 2007)
}

\begin{abstract}
We investigate the integer quantum Hall system in a two dimensional lattice model with spatially correlated disorder by using the efficient method to calculate the Chern number proposed by Fukui et al. [J. Phys. Soc. Jpn. 74, 1674 (2005)]. Distribution of charge density indicates that the extended states at the center of each Landau band have percolating current paths, which are topologically equivalent to the edge states that exist in a system with boundaries. As increasing the strength of disorder, the floating feature is observed in an averaged Hall conductance as a function of filling factor. Its relation to the observed experiments is also discussed.
\end{abstract}

DOI: 10.1103/PhysRevB.76.132202

PACS number(s): 73.43.-f, 71.30.+h, 71.55.Jv

Two dimensional electron systems with disorder in a perpendicular magnetic field have attracted much attention both experimentally and theoretically. According to the scaling theory, ${ }^{1}$ all single-electron states are localized at zero temperatures (Anderson localization). However, in the strong magnetic field, all states form Landau levels, and there exist delocalized states at the center of each Landau level, which are extended and formed percolating paths in a context of network model. ${ }^{2,3}$ The transition from the Hall liquid to the Anderson insulator in the limit of $B \rightarrow 0$ was discussed by Khmelnitskii and Laughlin in terms of the levitation scenario, i.e., floating theory, ${ }^{4,5}$ where Landau bands merge together as decreasing the magnetic field, and the extended states float up toward higher energy through the Fermi level. This scenario gives a reasonable explanation for many experiments, and the global phase diagram was constructed based on it. ${ }^{6}$ However, there still remain many controversial arguments about the microscopic picture of the floating theory. $^{7}$

In the quantum Hall system, the topological structure of the ground state wave function plays an essential role for quantization of the Hall conductance. Each quantum Hall state can be assigned a topological quantum number as the Chern number, ${ }^{8}$ which is the Hall conductance in units of $e^{2} / h$. Although there are several works ${ }^{9-13}$ to calculate the Chern numbers and succeeded to explain experimentally observed direct transition, the random potential, which is spatially uncorrelated, is not compatible with the continuum limit. ${ }^{14,15}$ Koschny et al. proposed that unless the random potential has long range correlation, the floating theory in a lattice system cannot be consistent with that in a continuous system. ${ }^{14}$ By investigating the localization length using a recursive Green-function method, ${ }^{16}$ they found that the negative-Chern number cannot be created if the correlation length of randomness is long enough. However, since the main part of their study is to calculate the Green function, the topological nature of extend states is unclear.

To study the topological nature of one-electron states, we numerically calculate both the spatial distribution of wave functions and the Chern number in this Brief Report. Here, we make a spatially correlated random potential in order to explore features of a continuous system in a lattice system. In previous studies on the floating scenario with the correlated disorder, ${ }^{14,15,17-19}$ the connection between the spatial probability density and the Chern number has not been focused on. To calculate the Chern number, an efficient numerical method recently proposed by Fukui et $a l^{20}$ is employed.

A tight-binding Hamiltonian on a square lattice with nearest neighbor hopping is given by

$$
H=t \sum_{\left\langle r, r^{\prime}\right\rangle} c_{r}^{\dagger} e^{-i \theta_{r, r^{\prime}}} c_{r^{\prime}}+\text { H.c. }+\sum_{r} w_{r} c_{r}^{\dagger} c_{r} .
$$

The magnetic flux per plaquette $\phi$ is given by $\sum_{\text {plaquette }} \theta_{r, r^{\prime}}$ $=2 \pi \phi$, where the summation runs over four links around a plaquette. We choose the Landau gauge, that is, $\theta_{r, r+\hat{x}}=0$ and $\theta_{r, r+\hat{y}}=2 \pi \phi r_{x}$. We set the units of energy to $t=1$ and mostly set the magnetic field per one plaquette $\phi=1 / 16$. A random potential $w_{r}$ is spatially Gaussian correlated as

$$
\left\langle w_{r} w_{r^{\prime}}\right\rangle=W e^{-\left|r-r^{\prime}\right|^{2} / \lambda^{2}}
$$

with mean value $\left\langle w_{r}\right\rangle=0$, where $\langle\cdot\rangle$ denotes the ensemble average over different randomness realizations. The distribution of the random potential is characterized by the correlation length $\lambda$ and the strength $W$. Although the $\lambda$ dependence is important, ${ }^{18,19}$ we will focus on the dependence on $W$ in the present Brief Report, and $\lambda$ is fixed to a value large enough to consider a continuous system in a lattice system.

In order to calculate Hall conductance in a system without the translational symmetry, we impose a generalized boundary condition on every randomness realization by requiring $T_{\mu}\left(L_{\mu}\right)|\psi\rangle=\exp \left(i \theta_{\mu}\right)|\psi\rangle(\mu=x, y)$, where $T_{\mu}\left(L_{\mu}\right)$ is the magnetic translation operator for a displacement $L_{\mu}$ and $\theta_{\mu}$ is the boundary-condition phase. The system size is $L=L_{x} \times L_{y}$. The Hall conductance $\sigma_{x y}$ can be calculated by $\boldsymbol{\theta}$ dependence of the wave function, ${ }^{8,21}$ where $\boldsymbol{\theta}$ is a vector defined as $\boldsymbol{\theta}=\left(\theta_{x}, \theta_{y}\right)$. As for fixed particle density, we define a ground state multiplet $\boldsymbol{\psi}(\boldsymbol{\theta})$, which is a $L \times q$ matrix written as $\boldsymbol{\psi}(\boldsymbol{\theta})=\left(\left|\psi_{1}(\boldsymbol{\theta})\right\rangle, \ldots,\left|\psi_{q}(\boldsymbol{\theta})\right\rangle\right)$ with $H(\boldsymbol{\theta})\left|\psi_{j}(\boldsymbol{\theta})\right\rangle=\epsilon_{j}(\boldsymbol{\theta})\left|\psi_{j}\right\rangle .{ }^{22}$ Here, $q$ is a number of one-particle states below the Fermi energy. Then, we define a non-Abelian Berry connection $\mathcal{A}$ as $\mathcal{A}=\boldsymbol{\psi}^{\dagger} d \boldsymbol{\psi}$. Finally, the associated Chern number is given by 


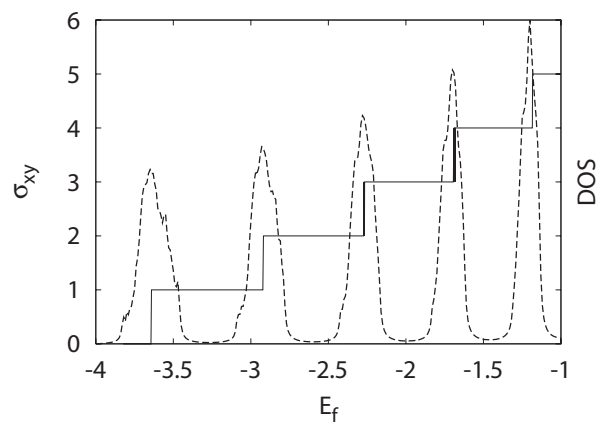

FIG. 1. The Hall conductance $\sigma_{x y}$ (solid line) as a function of Fermi energy $E_{F}$, and density of states versus energy $E$ (dashed line), at a lattice size of $32 \times 32$. The magnetic flux per plaquette $(\phi)$ is $1 / 16$, the correlation length $(\lambda)$ is 8 , and the disorder strength $(W)$ is 0.01 .

$$
C_{\psi}=\frac{1}{2 \pi i} \int_{S} \operatorname{Tr} d \mathcal{A},
$$

where $S$ is a two dimensional torus parametrized by the boundary-condition phases $\theta_{x}$ and $\theta_{y}$. Recently, Fukui et al. ${ }^{20}$ developed an efficient method to calculate a Chern number on a discrete momentum space by applying the geometrical formulation of topological charges in the lattice gauge theory. As an advantage of their method, one can obtain the Chern number accurately even if one uses coarsely discretized mesh points in the momentum space.

When randomness is introduced to a pure system, the Landau bands will broaden. At the tails of each band, all one-electron states become localized due to the Anderson localization. On the other hand, there still exist extended states at the center of each Landau band. Figure 1 shows the dependence of Hall conductance $\sigma_{x y}$ and density of states (DOS) on the Fermi energy $E_{F}$ for the given randomness realization that is plotted in Fig. 2(a) as a contour map. The strength of the randomness $W$ is set to 0.01 . The energy gap induced by the randomness is small. Nevertheless, numerical calculations of the Hall conductance are stable. As shown in Fig. 1, the Hall conductance jumps whenever the Fermi energy goes across the center of each Landau band. The extended states in the center of each Landau band can be classified into two classes: one possess, nonzero Chern number, which is called a critical state, and the other does not. There is only one critical state in the middle of the lowest band as well as the second lowest band. In general, there can exist several critical states at the center of the Landau band.

To study the topological structure of a wave function, the contour maps of the probability densities for several states are shown in Figs. 2(b)-2(d). Note that we adopted the periodic boundary condition. As shown in Fig. 2(b), the probability density of the localized state in the lowest band tail is concentrated around the valley of the random potential. On the other hand, the critical state, as seen in Fig. 2(c) or 2(d), forms a percolating path along the $y$ direction, which is associated with the percolation of equipotential lines., ${ }^{2,3}$ As shown in Fig. 2(d), two percolating paths and one nodal line between them are observed for the second Landau bands.
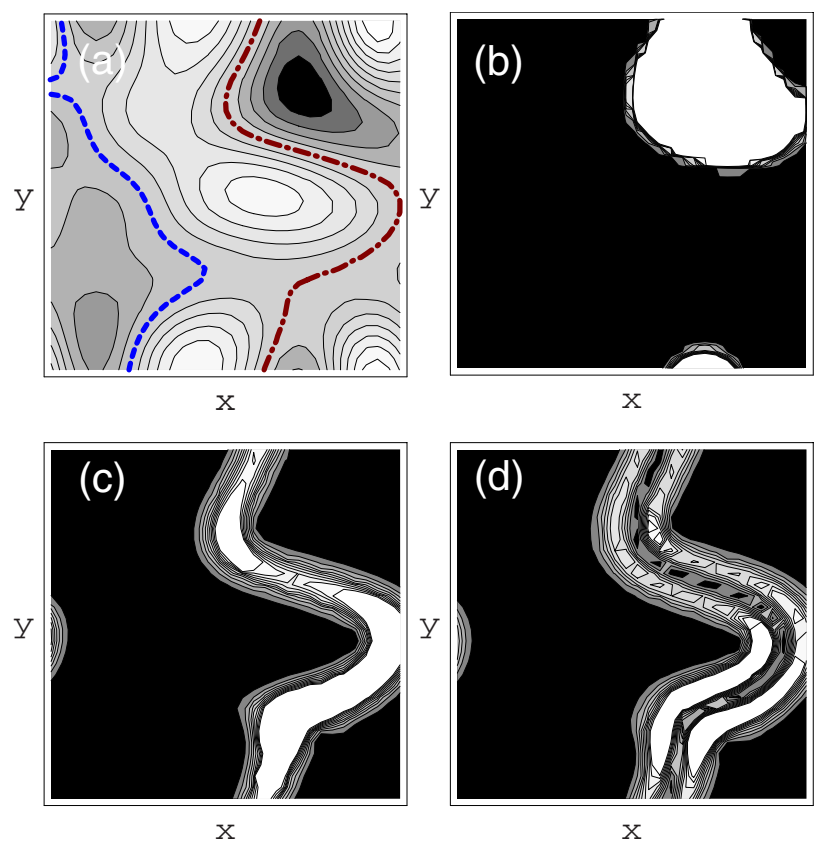

FIG. 2. (Color online) Contour plot of randomness realization for $\lambda=8$ (a). (b) The density probability distribution of several states, the localized state in the tail of lowest Landau band, (c) the critical state in the lowest Landau band, and (d) the critical state in the second lowest Landau band. The contour line indicated with the dashed-dotted line in (a) corresponds to the energy of the state of (c). The dotted line in (a) is the contour line with the same energy.

This distribution reminds us that the wave functions in the Landau gauge can be described by the Hermite function $H_{n}(r)$, which has $n-1$ nodal lines. Since other extended states which do not have nonzero Chern number show a similar spatial distribution, the percolating path is thought to be a characteristic feature of extended states.

There is a relationship between the topological character and the spatial distribution of wave function. Let us consider adiabatic processes of inserting the Aharonov-Bohm (AB) flux into the holes of the torus (see Fig. 3). Here, it should be noted that the topology of the square lattice used in the present Brief Report is a torus due to the periodic boundary condition. The penetration of flux $\Phi_{0}\left(\Phi_{1}\right)$ into the hole inside (outside) of the torus, which is equivalent to twisting the boundary phases $\theta_{y}\left(\theta_{x}\right)$, could induce an infinitesimal electric field in the $x(y)$ direction. The charge-current response to the electric field is given by the Byers-Yang formula:

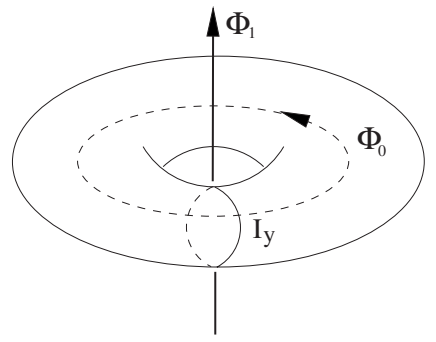

FIG. 3. Two possible insertions of Aharonov-Bohm flux through a torus. 

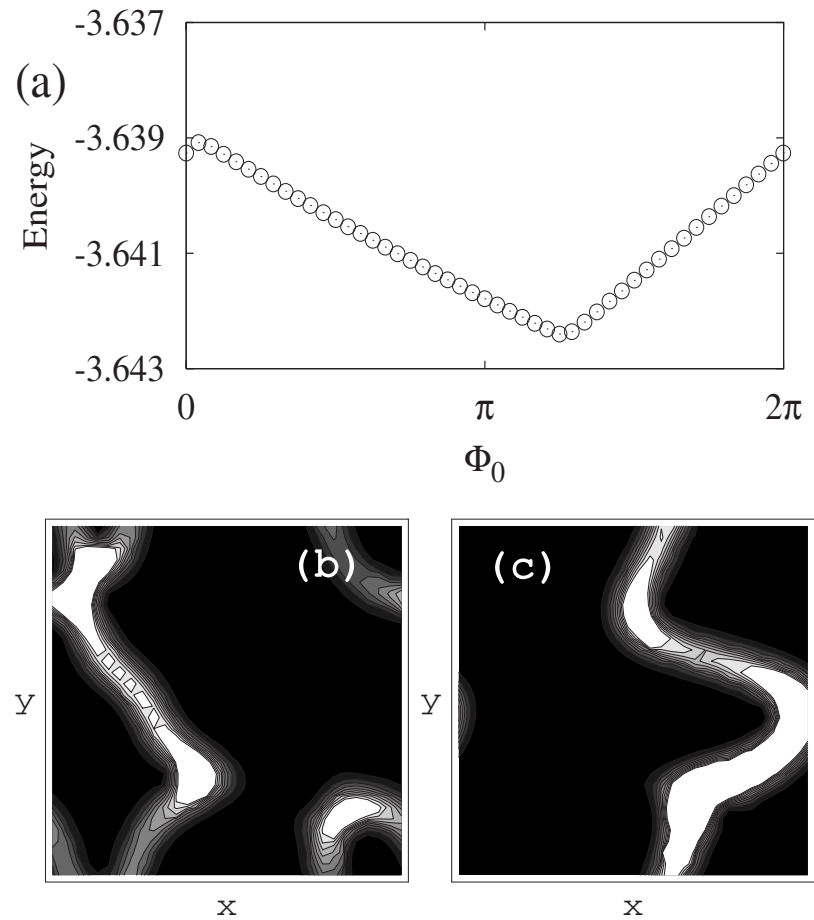

FIG. 4. (a) Energy dependence on AB flux. The distribution of probability density of the critical state in the lowest band when (b) $I_{y}>0$ and when (c) $I_{y}>0$.

$$
I_{x}=\frac{\partial E}{\partial \Phi_{0}}, \quad I_{y}=\frac{\partial E}{\partial \Phi_{1}},
$$

where $E$ is the total energy of the system.

When the $\mathrm{AB}$ flux in the unit of the magnetic flux quantum $h / e$ is adiabatically turned on from 0 to $2 \pi$, the spatial distribution of the probability density changes drastically, depending on the sign of $I_{y}$, as shown in Fig. 4. Figure 4(a) shows the energy as a function of the $\mathrm{AB}$ flux $\Phi_{0}$ for the critical state in the lowest band. Figure 4(b) or 4(c) shows the typical probability density when the energy increases (decreases) monotonously, i.e., $I_{y}>0\left(I_{y}<0\right)$. It turns out that this probability density corresponds to the percolating path along which the current flows. Actually, Fig. 4(b) or 4(c) corresponds to the contour line shown as the dotted line (or the dashed-dotted line) in Fig. 2(a). These two contour lines, which correspond to the percolating paths, indicate the same energy in the random potential. While the $\Phi_{0}$ dependence is drastic, we can hardly find a variation with $\Phi_{1}$ in the total energy and the distribution of probability density. This insensitivity to the flux $\Phi_{1}$ is attributed to the fact that there is no percolating path in the $x$ direction. We also found that the localized state is insensitive for both $\Phi_{0}$ and $\Phi_{1}$ because there is no current path in the $x$ direction or in the $y$ direction. The correspondence between the percolation path and the direction of the current can be understood in the view of a classic picture. In the system with boundaries, the electrons form skipping orbits along the boundaries corresponding to the cyclotron motion. The boundaries in this picture are formed by mountains of the random potential, which correspond to the dotted and dashed-dotted lines in Fig. 2(a). In
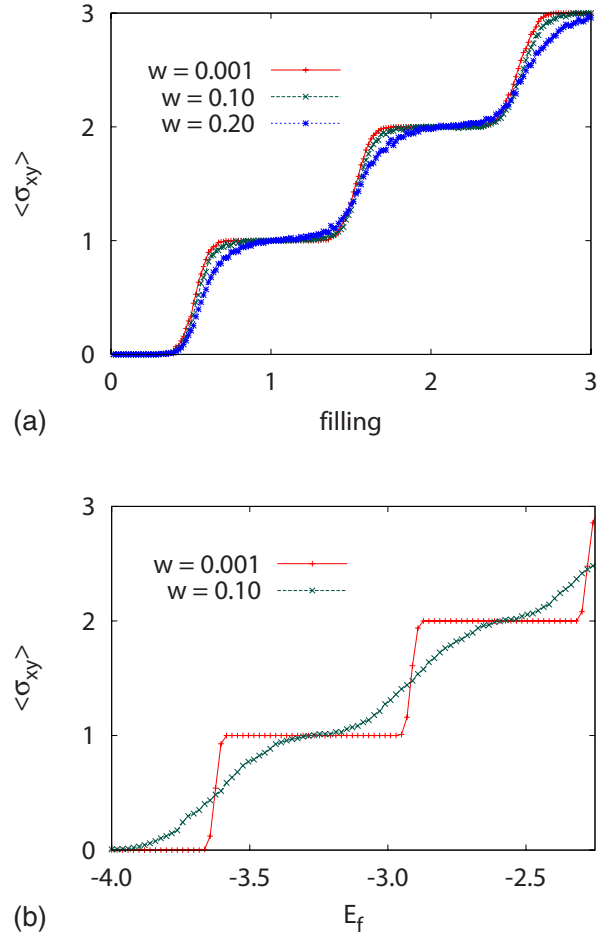

FIG. 5. (Color online) (a) The averaged $\sigma_{x y}$ as a function of filling factor and (b) Fermi energy $E_{F}$ at various strength of randomness. The correlation length $(\lambda)$ is 8 . The assemble average is taken by 320 randomness realization.

this meaning, the percolation paths are thought to be the edge states.

To take into account the experimental situation, we consider averaged Hall conductance $\left\langle\sigma_{x y}\right\rangle$, which is defined as an average over different randomness realizations. The effect of increasing the disorder strength $W$ on $\left\langle\sigma_{x y}\right\rangle$ as a function of the filling factor is shown in Fig. 5(a). With increasing $W$, one can see the broadening effect on the steps of the Hall conductance. Moreover, the step in the lowest Landau band systematically shifts to the higher filling. This behavior is consistent with the floating picture. On the other hand, the behavior of $\left\langle\sigma_{x y}\right\rangle$ as a function of energy is entirely different, as shown in Fig. 5(b). Sharp steps at a weak randomness $W=0.001$ are broadened completely by randomness in Fig. 5(b). This is because the randomness is not only broadening the Landau bands but also shifts the position on Landau bands depending on the mean value of random potential in each realization. This suggests that it is difficult to detect the float up in energy when the sample measured in experiment has many domain walls.

In conclusion, we have numerically calculated the Hall conductance, i.e., the Chern number, on a two-dimensional system with a uniform magnetic field and a spatially correlated random potential. We have found that a numerical method proposed by Fukui et al. is suitable for obtaining the Chern number in a system with a random potential. We have also investigated the spatial distribution of the wave functions for the study of the topology of extended states and 
localized states. The probability density of the extended states at the center of the Landau bands forms a current path associated with the percolation of equipotential lines. On the other hand, for the localized states in band tails, the charge density accumulates around the valley (or mountain) of the potential. The topological difference of wave function results in the different responses to the $\mathrm{AB}$ flux: the energy of the extended state is sensitive to the $\mathrm{AB}$ flux, while the $\mathrm{AB}$ flux has almost no effect on the localized state. Finally, after taking an average of the Hall conductance over different ran- domness realizations, floating feature to higher filling is observed.

This work was supported by Grant-in-Aid from the Ministry of Education, (No. 17540347) from JSPS, (No. 18043007) on Priority Areas from MEXT, and the Sumitomo Foundation. The computation has been partly done using the facilities of the Supercomputer Center, University of Kyoto and the facilities of the Supercomputer Center, Institute for Solid State Physics, University of Tokyo. *hatsugai@sakura.cc.tsukuba.ac.jp

${ }^{1}$ E. Abrahams, P. W. Anderson, D. C. Licciardello, and T. V. Ramakrishnan, Phys. Rev. Lett. 42, 673 (1979).

${ }^{2}$ S. A. Trugman, Phys. Rev. B 27, 7539 (1983).

${ }^{3}$ B. Kramer, T. Ohtsuki, and S. Kettemann, Phys. Rep. 417, 211 (2005).

${ }^{4}$ D. E. Khmelnitskii, Phys. Lett. 106A, 182 (1984).

${ }^{5}$ S. Kivelson, D. H. Lee, and S.-C. Zhang, Phys. Rev. B 46, 2223 (1992).

${ }^{6}$ R. B. Laughlin, Phys. Rev. Lett. 52, 2304 (1984).

${ }^{7}$ F. D. M. Haldane and K. Yang, Phys. Rev. Lett. 78, 298 (1997).

${ }^{8}$ D. J. Thouless, M. Kohmoto, M. P. Nightingale, and M. den Nijs, Phys. Rev. Lett. 49, 405 (1982).

${ }^{9}$ H. Aoki and T. Ando, Solid State Commun. 38, 1079 (1981).

${ }^{10}$ K. Yang and R. N. Bhatt, Phys. Rev. Lett. 76, 1316 (1996).

${ }^{11}$ Y. Hatsugai, K. Ishibashi, and Y. Morita, Phys. Rev. Lett. 83, 2246 (1999).
${ }^{12}$ D. N. Sheng and Z. Y. Weng, Phys. Rev. Lett. 78, 318 (1997).

${ }^{13}$ D. N. Sheng and Z. Y. Weng, Phys. Rev. Lett. 80, 580 (1998).

${ }^{14}$ Th. Koschny, H. Potempa, and L. Schweitzer, Phys. Rev. Lett. 86, 3863 (2001).

${ }^{15}$ D. N. Sheng, Z. Y. Weng, and X. G. Wen, Phys. Rev. B 64, 165317 (2001).

${ }^{16}$ A. MacKinnon and B. Kramer, Phys. Rev. Lett. 47, 1546 (1981).

${ }^{17}$ A. L. C. Pereira and P. A. Schulz, Phys. Rev. B 66, 155323 (2002).

${ }^{18}$ Th. Koschny and L. Schweitzer, Phys. Rev. B 67, 195307 (2003).

${ }^{19}$ Th. Koschny and L. Schweitzer, Phys. Rev. B 70, 165301 (2004).

${ }^{20}$ T. Fukui, Y. Hatsugai, and H. Suzuki, J. Phys. Soc. Jpn. 74, 1674 (2005).

${ }^{21}$ Q. Niu, D. J. Thouless, and Y. S. Wu, Phys. Rev. B 31, 3372 (1985).

${ }^{22}$ Y. Hatsugai, J. Phys. Soc. Jpn. 73, 2604 (2004); 74, 1374 (2005). 